\title{
Cardiac toxicity predictions: Safety pharmacologists correlate with the CiPA model
}

\author{
Hitesh B. Mistry ${ }^{1} \&$ Jaimit Parikh ${ }^{2}$ \\ ${ }^{1}$ Division of Pharmacy, University of Manchester, UK \\ ${ }^{2}$ IBM Research Center, New York, USA
}

\begin{abstract}
There has been a lot of interest and publicity regarding the use of a complex biophysical model within drug development for predicting the TdeP risk of new compounds. Throughout the development of the complex model numerous groups have shown that a simple linear mechanistic model explains the predictive behaviour of complex mechanistic models. That is the input-output relationship is almost linear even when complex kinetic assays are used. We hypothesized that given this linear relationship that scientist would be able to predict the outcome of the biophysical model. The objective of this pilot study was to assess the feasibility of such an analysis but also assess the initial degree of correlation. A set of 15 compounds with diverse ion-channel blocking against 4 ion-channel currents, IKr, ICaL, INa and INaL, was generated. Safety pharmacologists across numerous companies were approached and asked to categorize the TdeP risk of these compounds using only the \% block depicted via a bar chart into one of 3 categories: Risk, No-risk or Unsure. 12 scientists participated in the study, of which 11 correlated strongly with the model (11 person ROC AUC range: $0.86-1,7$ scientists had a value $>0.9$ ). The combined prediction of all scientists also correlated strongly with the model. These results highlight that the linear input-output relationship can indeed be predicted by the scientist. A future study exploring the degree of correlation with a wider group of scientists and wider set of compounds would be required to get a more precise estimate of the correlation. We hope this initial exploratory study will encourage the community to pursue this idea.
\end{abstract}




\section{Introduction}

There has been a lot of interest and publicity regarding the use of a complex biophysical model within drug development for predicting the TdeP risk of new compounds[1, 2, 3]. Throughout the development of the complex model numerous groups have shown that a simple linear mechanistic model explains the predictive behavior of complex mechanistic model $[4,5,6,7,8,9]$. That is the input-output relationship is almost linear even when complex kinetic assays are used. Indeed, this has been confirmed as a property of the CiPA in-silico model [10] via a global sensitivity analysis [11]. If many analyses have shown the input-output relationship to be linear [12,11]; Is a model actually required? We hypothesized that given this linear relationship that scientist would be able to predict the outcome of the biophysical model. Hence, we designed the following initial exploratory study to assess the strength of the correlation between scientist and the CiPA model predictions.

\section{Methods}

A set of 15 compounds with diverse ion-channel blocking against 4 ion-channel currents, IKr, ICaL, $\mathrm{INa}$ and INaL, was generated (Figure 1). Safety pharmacologists across numerous companies were approached and asked to categorize the TdeP risk of these compounds using only the \% block depicted via a bar chart into one of 3 categories: Risk, No-risk or Unsure. The correlation between Qnet, a metric derived from of the CiPA model response [10], and the scientist's choice was done in the following way.

For each scientists' predictions an ANOVA was conducted with the F-statistic and p-value reported. We also assessed whether Qnet correlated with Risk versus No-Risk/Unsure via the calculation of a ROC AUC reported. In addition, we also grouped all scientists' predictions by summing up their predictions for each compound, taking the average and comparing this with Qnet values.

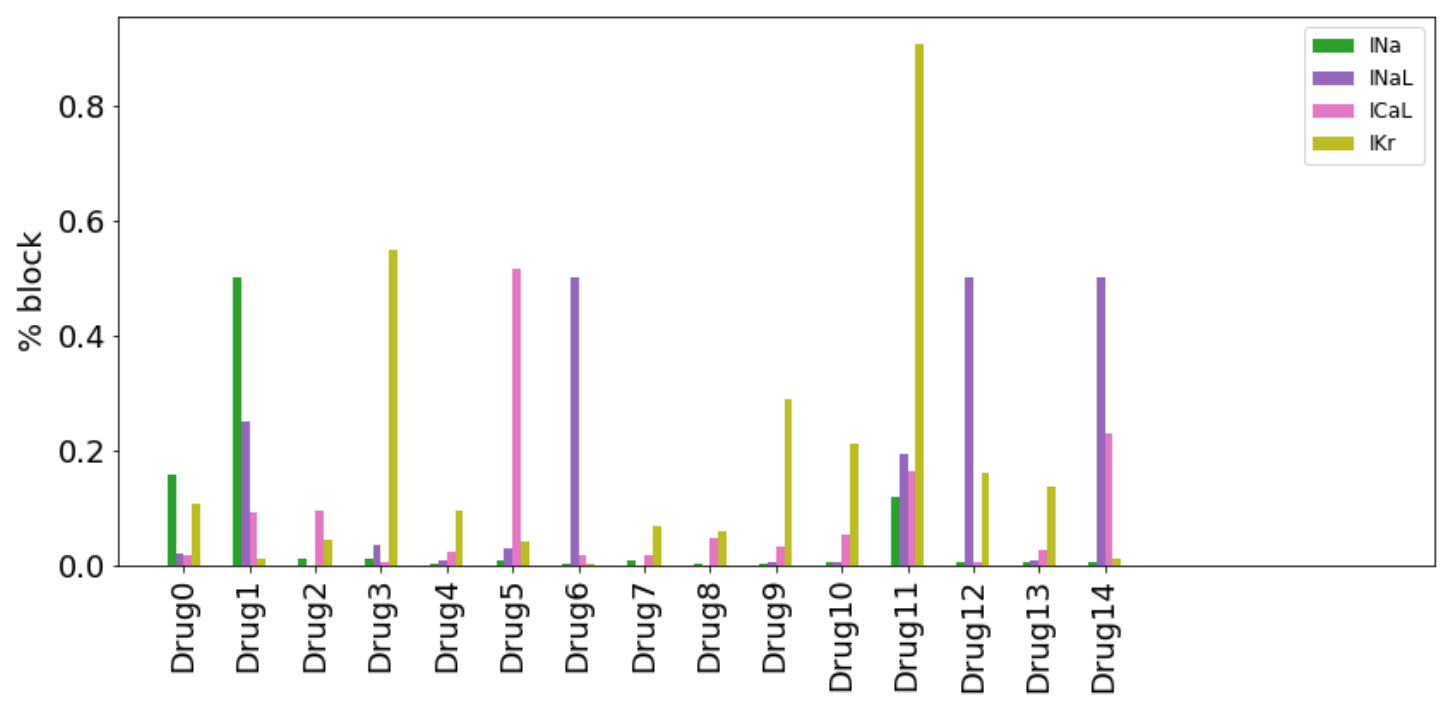

Figure 1: Plot showing the \%block of IKr, ICaL, INa and INaL channels for the 15 compounds.

\section{Results}

\section{Compounds}

Figure 2 shows the distribution of Qnet across the compound set and the thresholds for the CiPA categorization scheme. The plot shows that the compound set is quite diverse. 


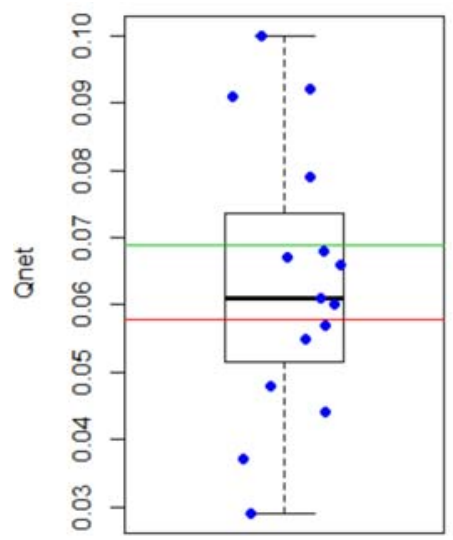

Figure 2: Plot showing the distribution of Qnet values across the compound set. Horizontal lines are the cut-off values from the CiPA validation study.

\section{Scientist/Model Correlation}

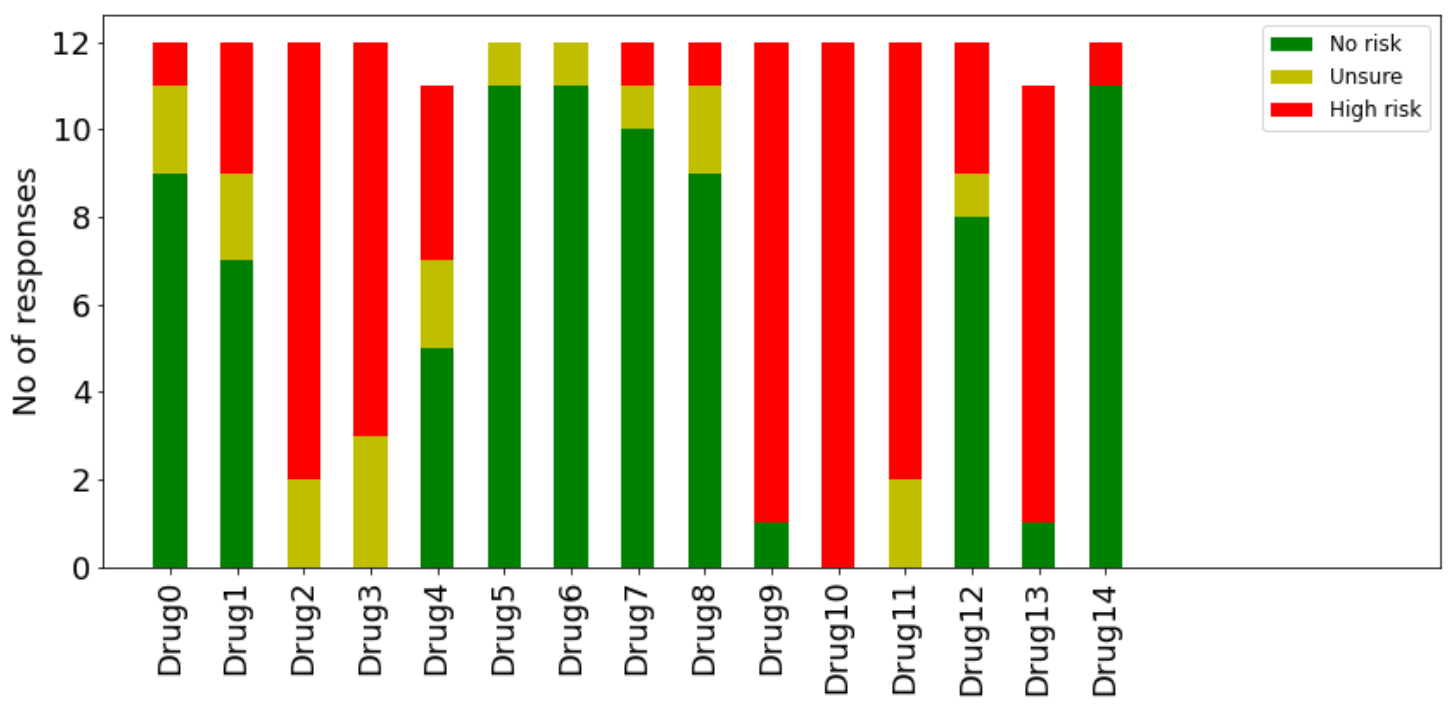

Figure 3: Plot showing the number of responses across different categories by the scientists for each of the 15 drugs.

Figure 3 shows the response of all scientists across each category for the 15 compounds. Table 1 shows that all scientists except one correlate strongly with Qnet via the two approaches. When we look at consensus across the scientists a strong correlation is found, see Figure 4.

Table 1: Individual scientist results

\begin{tabular}{|l|l|l|}
\hline Scientist & Risk vs & Risk vs \\
& Unsure vs No- & Unsure/No- \\
\hline
\end{tabular}




\begin{tabular}{|l|c|c|}
\hline & $\begin{array}{l}\text { Risk: } \\
\text { F-Statistic }(P- \\
\text { value) }\end{array}$ & $\begin{array}{l}\text { Risk: } \\
\text { ROC AUC }\end{array}$ \\
\hline 1 & $19.5(<0.001)$ & 0.98 \\
\hline 2 & $20.8(<0.001)$ & 0.96 \\
\hline 3 & $13.3(0.002)$ & 0.90 \\
\hline 4 & $20.8(<0.001)$ & 0.96 \\
\hline 5 & $12.8(0.004)$ & 0.86 \\
\hline 6 & $8.26(0.013)$ & 0.89 \\
\hline $\mathbf{7}$ & $\mathbf{0 . 0 2}(\mathbf{0 . 8 9 5})$ & $\mathbf{0 . 5 0}$ \\
\hline 8 & $5.33(0.038)$ & 0.84 \\
\hline 9 & $22.8(<0.001)$ & 1.00 \\
\hline 10 & $10.6(0.006)$ & 0.94 \\
\hline 11 & $25(<0.001)$ & 0.98 \\
\hline 12 & $10.6(0.006)$ & 0.86 \\
\hline
\end{tabular}

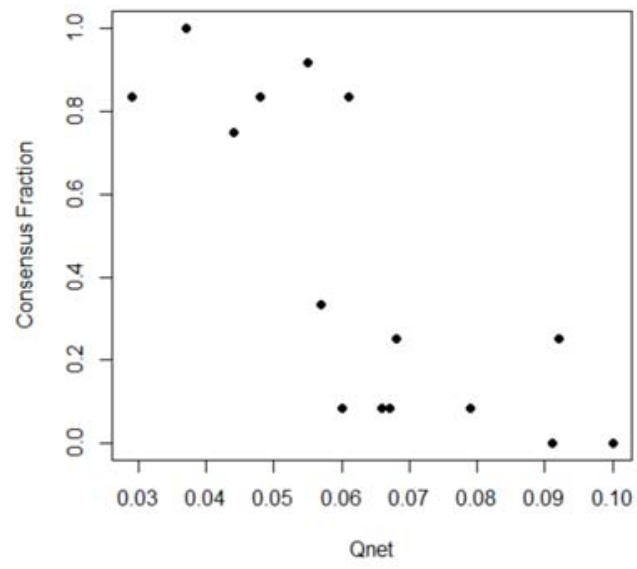

Figure 4: Plot showing the correlation between the Consensus Fraction and Qnet $(p=0.001)$

\section{Discussion}

Numerous groups have shown that the input-output relationship for the prediction of TdeP using ionchannel pharmacology data is linear $[12,7,11,9]$. This raises the question as to whether a model is needed at all let alone a complex model. In this initial exploratory study, we have found that scientists predictions do correlate well with Qnet, a complex model metric. This was seen for all scientist questioned except one. We hypothesis that the one scientist whose values did not correlate likely misinterpreted the question: is there any cardiac risk? However, this may not be the case. One approach to guard against a poor predictor within a group is to take a groups prediction. In our study we found the group prediction via a naïve average did correlate with Qnet.

In summary, these results highlight that the linear input-output relationship can indeed be predicted by the scientist. A future study exploring the degree of correlation with a wider group of scientists and wider set of compounds would be required to get a more precise estimate of the correlation. We hope this initial exploratory study will encourage the community to pursue this idea.

\section{References}

[1] B. Fermini, J. C. Hancox, N. Abi-Gerges, M. Bridgland-Taylor, K. W. Chaudhary, T. Colatsky, K. Correll, W. Crumb, B. Damiano, G. Erdemli, G. Gintant, J. Imredy, J. Koerner, J. Kramer, P. 
Levesque, Z. Li, A. Lindqvist, C. A. Obejero-Paz, D. Rampe, K. Sawada, D. G. Strauss and J. I. Vandenberg, "A New Perspective in the Field of Cardiac Safety Testing through the Comprehensive In Vitro Proarrhythmia Assay Paradigm , A New Perspective in the Field of Cardiac Safety Testing through the Comprehensive In Vitro Proarrhythmia Assay Paradigm," Journal of Biomolecular Screening, vol. 21, pp. 1-11, 2016.

[2] Z. Li, B. J. Ridder, X. Han, W. W. Wu, J. Sheng, P. N. Tran, M. Wu, A. Randolph, R. H. Johnstone, G. R. Mirams, Y. Kuryshev, J. Kramer, C. Wu, W. J. Crumb and D. G. Strauss, "Assessment of an In Silico Mechanistic Model for Proarrhythmia Risk Prediction Under the CiPA Initiative," Clinical Pharmacology \& Therapeutics, vol. 0, 2018.

[3] M. C. Lancaster and E. A. Sobie, "Improved Prediction of Drug-Induced Torsades de Pointes Through Simulations of Dynamics and Machine Learning Algorithms, "Clinical Pharmacology and Therapeutics, vol. 100, pp. 371-379, 2016.

[4] J. Kramer, C. A. Obejero-Paz, G. Myatt, Y. A. Kuryshev, A. Bruening-Wright, J. S. Verducci and A. M. Brown, "MICE Models: Superior to the HERG Model in Predicting Torsade de Pointes," Scientific Reports, vol. 3, p. 2100, 2013.

[5] H. B. Mistry, M. R. Davies and G. Y. Di Veroli, "A New Classifier-Based Strategy for in-Silico lonChannel Cardiac Drug Safety Assessment," Frontiers in Pharmacology, vol. 6, 2015.

[6] H. B. Mistry, "Complex versus Simple Models: Ion-Channel Cardiac Toxicity Prediction," PeerJ, vol. 6, 2018.

[7] J. Parikh, V. Gurev and J. J. Rice, "Novel Two-Step Classifier for Torsades de Pointes Risk Stratification from Direct Features.," Frontiers in pharmacology, vol. 8, p. 816, 2017.

[8] S. Han, S. Han, K.-S. Kim, H.-A. Lee and D.-S. Yim, "Usefulness of Bnet, a Simple Linear Metric in Discerning Torsades De Pointes Risks in 28 CiPA Drugs," Frontiers in Pharmacology, vol. 10, 2019.

[9] E. Passini, O. J. Britton, H. R. Lu, J. Rohrbacher, A. N. Hermans, D. J. Gallacher, R. J. H. Greig, A. Bueno-Orovio and B. Rodriguez, "Human In Silico Drug Trials Demonstrate Higher Accuracy than Animal Models in Predicting Clinical Pro-Arrhythmic Cardiotoxicity," Frontiers in Physiology, vol. 8, p. 668, 2017.

[10] S. Dutta, K. C. Chang, K. A. Beattie, J. Sheng, P. N. Tran, W. W. Wu, M. Wu, D. G. Strauss, T. Colatsky and Z. Li, "Optimization of an In Silico Cardiac Cell Model for Proarrhythmia Risk Assessment," Frontiers in Physiology, vol. 8, 2017.

[11] J. Parikh, P. Di Achille, J. Kozloski and V. Gurev, "Global Sensitivity Analysis of Ventricular Myocyte Model-Derived Metrics for Proarrhythmic Risk Assessment.," Frontiers in pharmacology, vol. 10, p. 1054, 2019.

[12] E. A. Sobie, "Parameter Sensitivity Analysis in Electrophysiological Models Using Multivariable Regression," Biophysical Journal, vol. 96, pp. 1264-1274, 2009. 